May 21st. Pulse 92. He had expectorated rust-coloured sputum. Vomiting was for the first time produced by the mixture. It was omitted, but the pill was continued.

May 23rd. The pulse was 96 , soft and regular. Te complained of but little pain. There was some dulness and bronchophony at the lower part of the left lung. A large blister was ordered. The pills to be taken twice a day.

He steadily improved from day to day. The pills were stopped on the 25 th, only a slight effect having been produced on the gums. He was ordered effervescing saline, with ipe. cacuanha and squills.

May 27th. No crepitation could be heard, but slight dulness remained. Pulse 80 .

June 2nd. He had quite recovered from his chest-symptoms.

During the treatment the diet was regulated very much according to the feelings of the patient. In the early part of the disease there was no appetite for food; but as the symp. toms began to subside, beef-tea, nutritious broths, and any food that could be taken, was allowed. After the subsidence of the pneumonia, he had a return of pain in the leg and slight inflammation of the saphena vein, which remained thickened for some time, and prevented him walking well. By the beginning of July, almost all symptoms had disappeared; but on the 9th, he went to some races, walked about a good deal, drank rather freely, and next day was seized with severe inflammation of the saphena vein, with an erysipelatous blush all over the leg. Rest and fomentations relieved the urgent symptoms, but left a thickening in the course of the vein. This was removed by rubbing in mercurial ointment, and regulation of diet. $\mathrm{He}$ soon afterwards went to the sea-side. When he returned, no appearance of any thickening of the vein existed. His health has continued very good ever since.

[To be continued.]

\section{Britinal Commanications.}

\section{A FEW MINOR QUERIES IN MEDICAL S C I E N CE.}

By W. Hinds, M.D., Lecturer on Botanical Science at the Birmingham Midland Institute.

\section{I.-NARCOTICS IN INCIPIENT INSANITY.}

The editor of the British Medical Journal, in his relation with contributors, began his career with a most wise and practically useful postulate. He announced, week after week, that " brevity was the soul of medical writing, even more than of wit", until it was fully evident that many amongst his staff of contributors looked upon that aphorism as an admirable bit of irony. With a view to the practical resuscitation of that maxim, which I shall assume to have been intended in its literal sense, I shall endeavour to contribute a short paper now and then on some subject of more or less interest to every medical man. If these papers be not read, or of sufficient interest to be read by many, I shall at least endeavour to compress whatever I may be able to give into the smallest possible space.

My first query is-To what extent can we entirely suppress incipient insanity by a very early and free use of opium?

Now, my theory is that much may be done in this direction. The fact is more or less acknowledged, especially by those whose express functions are employed in the treatment of insanity ; but it is not, I think, so thoroughly recognised by the profession in general as it should be. Cases of incipient in sanity do really belong, and ought to be considered to belong, to the professsion in general. It is the profession in general who first meet with them, and it is they to whom the very earliest treatment should be considered to belong. It is to be feared that many practitioners feel so much anxiety to get these cases into an asylum, the place especially devoted to such cases, that they let slip sometimes an opportunity which can never be compensated for. The anxiety for such a removal is highly commendable and important; but it so happens that often, in spite of it, delays will occur, either through the wavering resolves of the patient's friends, or from other causes.
The hours that thus pass are often golden hours in relation to the welfare of the patient, and they are often lost for ever.

To those who may coincide with this view, the followin cases will be read with interest, as illustrating the princlple laid down :-

CASE I. William N., aged 22, a steel-roller, getting a salary of about five pounds weekly as a manager, and not always temperate in his habits or regular in his hours, became insane about two years ago, and was taken to an asylum, where he was confined for sixteen weeks. From that period, he remaine well until a month ago, when, after smoking much and losing his rest, he became talkative and rambling. He did not sleep well at night, and was either talking unconnectedly, or else jumping and capering about the room. He was very mis chievous if left a few moments, and would open the window and throw out his shirt and any other things at hand. At length he became nearly unmanageable.

I saw him on August 21, 1858, after he had been really ill about a week. When first seen, he was very chattering, merry and complaisant, rambling and unconnected in his conversation, but extremely compliant to any direction. His tongue was furred, and his appetite bad; no tremors were present He did not sleep at night, and, after dozing off a little, would wake, and begin to talk, mixing all sorts of matters up into a confused medley.

Four grains of powdered opium were ordered to be taken immediately, and a mixture of compound spirit of ammonia and tincture of hyoscyamus in frequent doses.

Aug. 22nd. He had not slept, and was getting worse. Three quarters of a grain of morphia were ordered to be taken im. mediately, and repeated at bedtime.

Aug. 23rd. He had had not more than an hour's sleep, and was very wild. His bowels were confined. An aperient of sulphate of magnesia and rhubarb was directed to be taken immediately; and, after the bowels should have been opened, three grains of morphia in two pills, with an interval of six bours.

Aug. 24th. He had very little sleep during the night, and the symptoms were aggravated. The bowels had freely acted. He was ordered to take six grains of morphia in four pills, with an interval of six hours, and a tonic mixture.

Aug. 25th. He had slept four hours; there was slight improvement. The pills-namely, six grains of morphia, as before, were repeated. The result was that he improved gradually from this date; that on the 27 th and 28 th be became quite rational, and made anxious inquiries as to his business during the period of his illness, and on September 7th he had fully recovered his health.

In the seven days from the 21st to the 27th of August, he had taken twenty-five grains and a half of hydrochlorate of morphis, four grains of opium, and about one drachm of extract of hyoscyamus, besides a little tincture.

CASE II. Eleazer H., aged 40, of industrious and very tem. perate habits, was brought to me in the middle of the night of the 14th of September, 1857. His wife came with him, for safety. The moment I got sight of him, he hurriediy pulled off his coat, turned up his shirt.sleeves-at first, somewhat to my consternation-and demanded to be largely bled. His agitation was extreme, and he could not rest a moment. He explained to me that he was not in his right mind, had given way to violent rage, and had had a serious quarrel with his wife.

The history of this case, which I afterwards learned, was as follows :-For several months he was irritable and nervous, and felt his health failing. At last, be began to get suspicious and jealous of his wife, but without the least reason. A night or two before I saw him, after getting a pint or two of beer, he felt much worse, and got into a violent rage: He became agitated and sleepless, and had not slept for many nights. On the day before, he had himself carefully put out of the way some heavy weapons from his room, feeling that he might do violence, even against his own will.

Seeing at once that he was labouring under great exhaustion and excitement of the nervous system, I quieted his feelings, told him that bleeding would do him injury, and gave him four grains of powdered opium and a mixture of ammonia and tincture of hyoseyamus.

Sept. 15th (next day). When I called to see him, I found he had not slept more than half an hour, was greatly agitated, and unable to lie quiet in bed. He was quite incoherent at times. The tongue was coated somewhat, and not at all tremulous; the pulse was slow, and about 78 ; the bowels were freely open. A grain and a half of morphia and a grain of tartar emetic, 
divided into two doses, were prescribed to be taken, with an interval of five or six hours, in addition to the mixture.

Sept. 16th. He had about an hour's sleep altogether. $\mathrm{He}$ dozed a little, and started up in a fright. $\mathrm{He}$ was slightly more composed. An aperient was given, and the morphia was increased to a grain and a half at each dose, with a grain of tartar emetic twice in the day.

Sept. 17th. He had not much sleep, but was more rational, the lucid intervals being more pronounced. He could now lie quite still. The bowels were well open. He was ordered to take two grains of morphia and three-fourths of a grain of ar.timony twice in the day.

Sept. 18th. He had four hours sleep. The pills were repeated.

Sept. 20th. The pills procured a fair night's sleep on the 18th. Last night he did not sleep so well. The narcotic seemed less active. He was ordered to take half a grain of morphia and five grains of extract of hyoscyamus every night.

From this date he improved rapidly, sat up on the 21st, and on the 2 (ith he went to business as usual. In eight days he took twenty grains of hydrochlorate of morphia and four grains of opium, besides some doses of hyoscyamus. Some time ago, he had had no subsequent attack.

I might give other cases in illustration of the principle; but the results in these two are so striking, evident, and conclusive, that it would not be either necessary or desirable to the object in view.

\section{ON THE GENERATION OF NERVE-FORCE.}

\section{By Richard Hugnes, M.R.C.S., and L.R.C.P.Ed.(Exam.),} Surgeon to the Brighton Orthopædic Hospital.

Thene is a tendency in much of modern science to revive upon sound scientific data the guesses of earlier speculators. 'The atomic theory in physics and chemistry, the reflex doctrine in nervous physiology, will supply examples of my meaning. It will not then be urged, as an objection to the theory I am about to advance, that it may be found adumbrated in the works of Harvey, Willis, and others.

It is generally admitted that neurine, or nervous matter, performs its peculiar functions in the organism by being the seat of a property which, from its analogy with the so-called "forces" of inorganic nature-light, heat, electricity, etc.-is called the nervous force. Farther, that, unlike the contractile property of muscle, which resides in every fibre as such, the gray or vesicular neurine is the generator, the white or tubular neurine the transmitter of this force. These doctrines are so generally accepted, that I may start with them as assumptions.

The common theory of the mode whereby this nerve-force is thus generated and transmitted in the motor process seems to be as follows:-Upon the accession of a stimulus, from within or from without, on the nervous centre, an evolution of force from the gray matter there takes place, which force is then transmitted through the tubular matter of the nerves to the muscles appropriate to the action desired. I would, on the contrary, propose the following hypothesis:-

That the nervous force is being continuously and uninterruptedly generated or secreted from the blood by the cells of the gray matter, and as constantly transmitted therefrom through the nerves; and that any stirnulus-whether this be volitional, emotional or reflex, galvanical or mechanical-produces contraction, not by causing evolution of nerve-force altogether de novo, but by increasing the intensity of the generation or transmission already going on.

There are three points in this hypothesis which require proof. (a) That the nervous force is generated by the gray matter from the blood; $(b)$ that its generation is continuous, not interrupted. If these be proved, another proposition(c) that motor stimuli act, not by producing nerve-force altogether de novo, but by intensifying processes already in transaction, will be manifest without farther argument.

$a .1$. In the first place, let us consider one of the main grounds whereon we believe the grey matter to be the generator, the the white matter the transmitter, of nerve-force. It is that the grey matter presents precisely the structure which in every gland of the body is characteristic of its secreting portion, viz., a collection of nucleated cells; while the tubular form of the white matter presents a no less perfect analogy to the ducts whereby the secretions are transmitted to their appropriate destinations (Solly, On the Brain,p.1-17). But in every gland there is, besides secreting cells and transmitting tubes, a third constituent, viz., the fluid from which the particular secretion is formed. Is the analogy here to fail? Are the nucleated cells of the nervous system to be regarded as generating their peculiar secretion from nothing, or at best from themselves? Shall we not rather say that the blood-the source of everything else in the body, even of the future progeny*-is to this, as to other glands, the material on which its secernent function works?

2. What are the grounds on which, whatever may be our view of the peculiar function which the spleen plays in the organism, we are at least certain that it has some office to perform in reference to the blood? Simply that there is conveyed to it a far larger quantity of blood than it requires for its nutrition. We argue that it must do something, though we may not know what, with this excess of supply. Now the amount of blood conveyed to the brain is enormously beyond that required for its mere nutrition, being not less than onefifth of the total amount contained in the body; while the weight of the brain in proportion to that of the body is but one-thirty-fifth. And of this blood by far the greater quantity goes to the grey matter, which nevertheless itself exists in the brain in a smaller proportion than the white matter. When, added to this, we have, what in the spleen we have not, a system of nucleated cells undoubtedly generating nerve-force, and a system of tubular nerves undoubtedly transmitting it to its destination, can we doubt that this force is secreted from the largely-superabundant supply of blood ?

3 . The state of unconsciousness (syncope) which almost immediately follows on the diminution to any great extent of the supply of blood to the brain, is explicable upon no hypothesis so well as upon that which supposes that from the blood that force is continuously generated which is the mind's instru. ment, and without which it has no consciousness of anything external to itself. For then any interruption, however temporary, to the supply of blood would be shortly followed by unconsciousness, which could not be the case for a much longer time were the loss of nutrition the only result.

In thus speaking of nerve-force as a secretion from the blood, I am by no means committing myself to any theory as to the mode of its production, or as to its nature as a material though imponderable agent. Its analogue in the inorganic world seems to be electricity; and it is by no means necessary to suppose this a material fluid, although we speak of it as generated (or set free) by chemical decomposition and other changes.

$b$. My second proposition is that this generation of nerveforce is continuous, not interrupted. In support of this I may adduce the following fact:-If a motor nerve be divided transversely, in a living animal, and the distal portion be galvanically or mechanically irritated, vigorous contractions are produced in the muscles supplied by that nerve. The same result follows on irritation for some time after the section, but in a gradually decreasing intensity, until it altogether ceases to be manifested upon any amount of stimulus. Now this change is not to be explained by supposing any loss of vitality on the part of the distal portion of the nerve ; for its supply of blood is in no way cut off by the section, and, upon re-union of the divided ends taking place, its function is completely restored; the only altered circumstance is that its connection with the grey matter of the nervous centres has been interrupted. Its loss of excitability simply from this cause would be perfectly intelligible upon the old hypothesis; but not so the presence of excitability immediately after the section, and its gradual decrease and extinction afterwards. The hypothesis I propose will perfectly explain all the phenomena. Nerve-force is being continually generated in the nervous centres, and transmitted through the nerves, so that the latter are kept constantly charged as it were with it; and an external stimulus applied at any part of their course will excite them to produce muscular contraction. Being constantly charged with nerve-force, a separation from the nervous centre at first induces no change in their excitability; but, their connection with the fountain being cut off, their supply of force is gradually exhausted, till at length they become inert cords of nervous matter, insensible to the strongest stimuli.

The preceding explanation of this phenomenon is confirmed by observing the mode in which this exhaustion of excitability takes place, viz., that it does not affect the whole

* For the testis and the ovary can hardly be regarded in any other light than as two glands, secreting from the blood (of an homologous artery, spermatic or ovarian), the one the sperm-cell (spermatozoon), the other the germ-cell (ovum), whose union constitutes the future progeny. 